\title{
LA CIRCULACION MONETARIA EN LOS TERRITORIOS INTERIORES DEL NORTE DE LA PENINSULA IBERICA, DURANTE LOS PRIMEROS AÑOS DEL ALTO IMPERIO ROMANO
}

\author{
MANUEL MEDRANO MARQUES
}

Nuestro objetivo primordial en este trabajo es analizar la circulación de moneda de bronce emitida entre 27 a. C. y $41 \mathrm{~d}$. C. en cuatro zonas relativamente próximas, pero de características socioeconómicas y culturales diferentes, situadas en el área septentrional de Hispania. Y ello, a lo largo de este período, en el cual se producen la máxima expansión y la extinción prácticamente total de las emisiones autónomas.

Los conjuntos cuya circulación monetaria va a ser analizada son cuatro (véase mapa "Conjuntos monetarios»): 1) Jalón, que se ha constituido con los hallazgos efectuados en varios lugares ubicados en los valles medio y alto de este río. 2) Ebro, formado con piezas procedentes de La Rioja y Alava. 3) Clunia, donde se contabilizan las monedas encontradas en esta ciudad meseteña. 4) León, integrado por numerario procedente del área de esta provincia que forma parte de la Meseta Norte. Estos conjuntos han servido también como base para realizar pruebas estadísticas, destinadas a estudiar el grado de similitud entre sus circulaciones monetarias.

Como hemos señalado, el material que se contabiliza dentro de cada uno de estos conjuntos fue acuñado, en su totalidad, entre el momento en que Octaviano tomó el título de Augusto (27 a. C.), dando comienzo a ia fase histórica denominada Alto Imperio romano, y la muerte de Calígula. Desde el punto de vista numismático, este período tiene un interés excepcional en la Península Ibérica. A lo largo de la primera mitad del siglo i a. C., fueron cesando buena parte de las emisiones autónomas hispanas, y todas las producciones de carácter ibérico. Ello debió producir, en la segunda mitad de este siglo, una situación de penuria monetaria, puesto que el taller de Roma no fue capaz de abastecer las necesidades del país. Para proveer adecuadamente de numerario a la Península, Augusto procedió a incrementar el número de cecas autónomas, reordenando las acuñaciones, posiblemente a la vez que llevaba a cabo la reorganización provincial de $27-13$ a. C. ${ }^{1}$.

\footnotetext{
'Beltrán (1986), p. 908.
} 
Esta nueva estructuración de la producción monetaria supuso que, la misma, fue confiada de forma prácticamente exclusiva a colonias y municipios. Durante la segunda mitad del reinado de Augusto, y el de Tiberio, se estabilizó la producción, e incluso se incrementaron bajo este último emperador los centros emisores en algunas zonas, como el valle medio del Ebro. Finalmente, Calígula, siendo consecuente con su política centralizadora, y siguiendo la tendencia iniciada por Augusto que pretendía reducir el número de talleres monetarios occidentales, procedió a cerrar las cecas hispanas, en dos etapas: inicialmente, clausuró todos los centros de producción de la Bética y Lusitania, y en la Tarraconense, de 19 cecas activas con Tiberio, mantuvo sólo siete. En torno al año $40 \mathrm{~d}$. C., o incluso antes, estos siete talleres fueron también cerrados, terminando así las emisiones autónomas hispanas, a excepción de la acuñación de Insula Augusta con el retrato de Claudio. Este fenómeno, que tuvo carácter general en Occidente, condujo a la total concentración en Roma de la amonedación de bronce de esta parte del Imperio ${ }^{2}$.

Algunas circunstancias históricas han podido determinar especialmente la evolución de las emisiones autónomas en la Tarraconense, así como la forma en que se produjo su dispersión. Particularmente, destaca el hecho de que buena parte de las guerras cántabras se desarrollase en los primeros años del Principado, hasta 19 a. C. Este conflicto supuso la presencia en el noroeste de la península de seis o siete legiones, con sus correspondientes tropas auxiliares, algunas de las cuales permanecerían en Hispania con posterioridad al cese de las hostilidades. De hecho, la drástica reducción del número de cecas que llevó a cabo Calígula, ha sido puesta en relación con la retirada de tropas de la Tarraconense por algunos autores ${ }^{3}$.

Otra circunstancia de gran relieve viene determinada por el hecho de que, sin lugar a dudas, el acceso a la economía monetaria fue un fenómeno que se produjo en las diversas zonas de Hispania de modo no simultáneo, lo que repercutió en el carácter de la circulación monetaria de los diferentes lugares. Evidentemente, en la región leonesa, por ejemplo, la moneda no debió constituir el elemento de referencia habitual en las relaciones económicas básicas, ni se dio una vida urbana intensa, durante buena parte del período que estudiamos.

Antes de pasar a establecer el inventario de hallazgos, debemos efectuar algunas precisiones acerca de los criterios que se han seguido a la hora de recopilar el material que constituye estos cuatro conjuntos. Como hemos señalado, vamos a considerar las monedas de bronce emitidas entre 27 a. C. y $41 \mathrm{~d}$.C., sean o no autónomas, aparecidas en las zonas que se analizan.

2 Beltrán (1986), pp. 911-913.

3 Beltrán y Beltrán (1980), p. 39. 


\section{CONJUNTOS MONETARIOS}

1: Jalón. 2: Ebro. 3: Clunia. 4: León

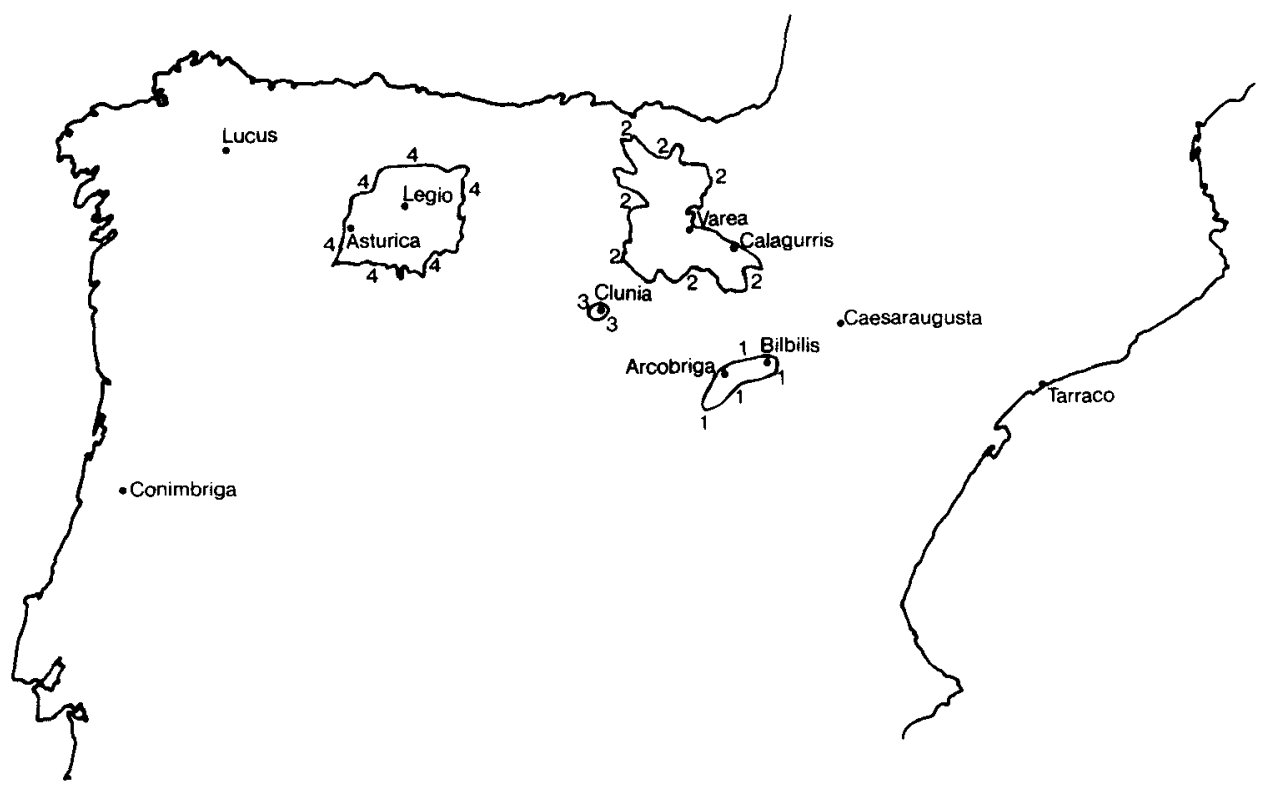

Excluimos las piezas de metal noble, porque su valor intrínseco tiene como consecuencia que su circulación se rija por criterios diferentes, siendo estas monedas mucho más propensas a fenómenos como la retirada de la circulación (atesoramiento), larga perduración cronológica, etc. Tampoco contabilizaremos los numismas procedentes de tesoros u ocultaciones, que pueden proporcionarnos informaciones distorsionadas por los procesos de selección, la prolongada vigencia temporal, etc. Finalmente, no se tendrán en cuenta las imitaciones de acuñaciones oficiales, extraordinariamente escasas $y$, muy probablemente, producidas con bastante posterioridad a los originales que copian.

A continuación, procederemos a exponer el modo en que hemos constituido cada uno de los conjuntos, así como el origen de sus datos. 


\section{VALLE DEL JALON}

Está formado por los hallazgos efectuados en la ciudad de Bilbilis y su entorno inmediato, dentro del valle medio del Jalón, así como por piezas procedentes de Arcobriga y Luzaga, lugares que se inscriben en el ámbito del Alto Jalón. Estos territorios tuvieron sin duda gran importancia como vía de acceso, desde el Valle del Ebro, a las Submesetas Norte y Sur de la Península Ibérica. Los datos se han extraído de las publicaciones de MartínBueno (1971 y 1973-74), Martín-Bueno y Redondo (1979), Domínguez y Galindo (1984), y Medrano, Diaz, Astiz y Lizabe (en prensa). Las monedas recogidas por Martín-Bueno y Redondo son de procedencia insegura, si bien parece muy probable que tengan su origen, mayoritariamente al menos, en la comarca de Calatayud ${ }^{4}$. En cuanto a los numismas publicados por Domínguez y Galindo, han sido hallados en campos de los alrededores de Cala-

\section{CUADRO 1}

Valle del Jalón

\begin{tabular}{|c|c|c|c|c|}
\hline & Augusto & Tiberio & Caligula & $\begin{array}{l}\text { Indeter- } \\
\text { minadas }\end{array}$ \\
\hline 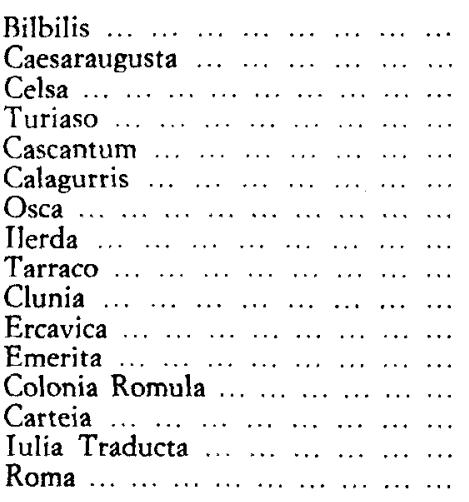 & $\begin{array}{r}9 \\
12 \\
9 \\
2 \\
3 \\
1 \\
1 \\
3 \\
1 \\
3 \\
3 \\
1\end{array}$ & $\begin{array}{l}2 \\
4 \\
1 \\
2\end{array}$ & 2 & 3 \\
\hline $\begin{array}{llllllll}\text { Total } & \ldots & \ldots & \ldots & \ldots & \ldots & \ldots & \ldots\end{array}$ & 48 & 23 & 5 & 3 \\
\hline $\begin{array}{lllllllll}\text { Porcentajes } & \ldots & \ldots & \ldots & \ldots & \ldots & \ldots & \ldots\end{array}$ & 60,76 & 29,11 & 6,33 & 3,80 \\
\hline
\end{tabular}

' Martín-Bueno y Redondo (1979), pp. 8-9. 


\section{VALLE DEL JALON}

Aporte porcentual por cecas

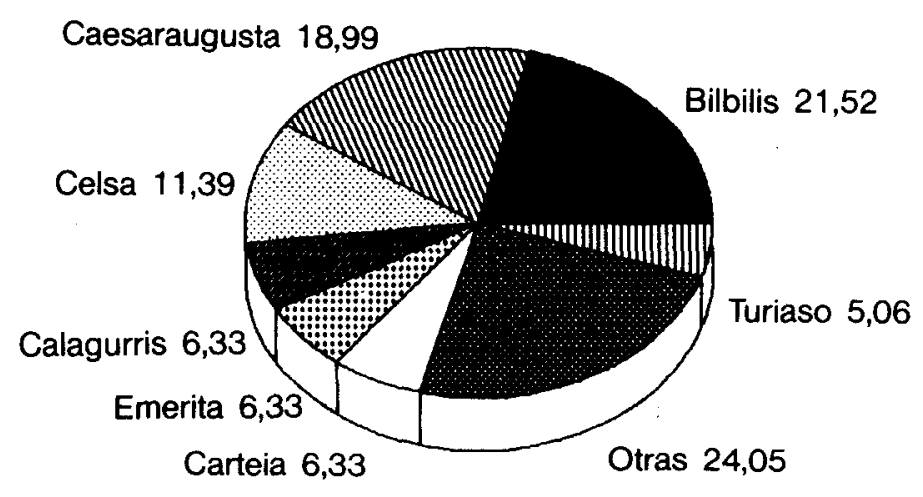

tayud, procediendo muchos de ellos del despoblado de Valdeherrera ${ }^{5}$. El inventario de las piezas de este conjunto figura en el cuadro 1, clasificado por cecas y emperadores.

\section{VALLE DEL EBRO}

Se ha formado este grupo a partir de monedas encontradas en las actuales provincias de La Rioja y Alava, territorios correspondientes al ámbito geográfico, económico y cultural del Valle del Ebro. Desde las vías que cruzan esta zona se accede también con relativa facilidad a las llanuras septentrionales de la Península. Los datos numismáticos han sido extraídos de los trabajos de Nieto Gallo (1958), Ugartechea (1967), Martín-Bueno (1974), Elorza (1974), Sáenz de Urturi (1978), Galve y Andrés (1984), Jiménez, Luezas, Sáenz e Iradier (1985), y Sáenz de Buruaga y Sáenz de Urturi (1986). Las piezas del Museo Provincial de Logroño presentadas por Martín-Bueno son todas de procedencia desconocida, y si bien parte de ellas parecen tener su origen en la región, el conjunto ofrece solamente una fiabilidad relativa ${ }^{6}$. Presentamos en el cuadro 2 la relación de hallazgos en esta zona.

${ }^{5}$ Dominguez y Galindo (1984), p. 63.

- Martín-Bueno (1974), pp. 65-66. 


\section{CUADRO 2}

\section{Valle del Ebro}

\begin{tabular}{|c|c|c|c|c|}
\hline & Augusto & Tiberio & Caligula & $\begin{array}{l}\text { Indeter- } \\
\text { minadas }\end{array}$ \\
\hline 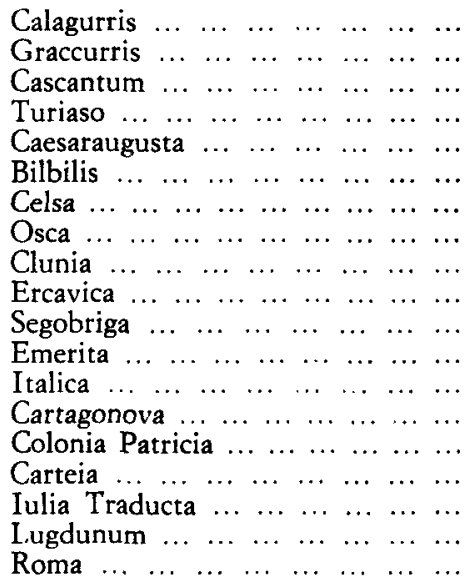 & $\begin{array}{l}6 \\
7 \\
5 \\
3 \\
1 \\
\\
5 \\
3 \\
4 \\
3 \\
4 \\
3\end{array}$ & $\begin{array}{l}6 \\
1 \\
2 \\
1 \\
2\end{array}$ & 1 & \\
\hline $\begin{array}{lllllllll}\text { TOTAL } & \ldots & \ldots & \ldots & \ldots & \ldots & \ldots & \ldots\end{array}$ & 51 & 19 & 2 & \\
\hline $\begin{array}{lllllllll}\text { Porcentajes } & \ldots & \ldots & \ldots & \ldots & \ldots & \ldots & \ldots\end{array}$ & 70,83 & 26,39 & 2,78 & \\
\hline
\end{tabular}

\section{VALLE DEL EBRO}

Aporte porcentual por cecas

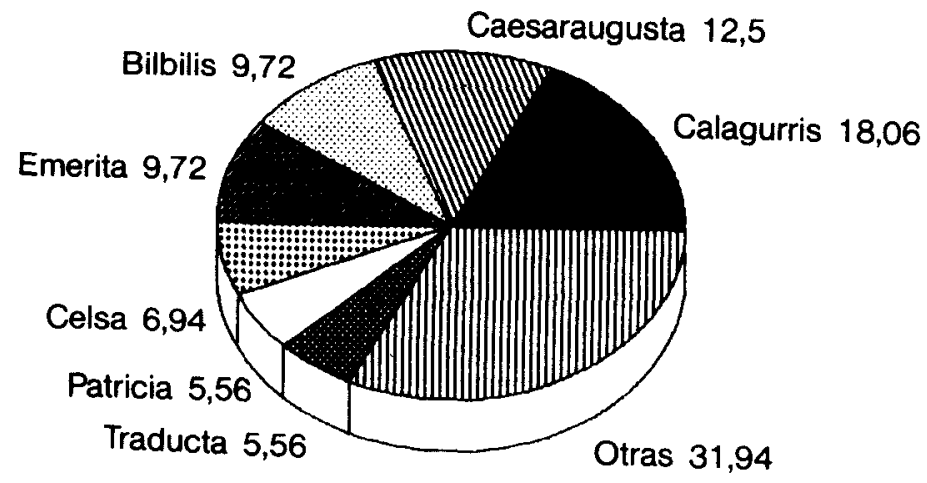




\section{CLUNIA}

Los numismas proceden de las excavaciones en la ciudad de Clunia, que se ubica no lejos de los territorios de los conjuntos anteriores, pero dentro del ámbito de la Submeseta Norte. Han sido publicados por Gurt Esparraguera (1985) en la monografía dedicada por este autor a la circulación monetaria en el núcleo urbano. En el cuadro 3 presentamos la clasificación de los hallazgos.

\section{CUADRO 3}

\section{Clunia}

\begin{tabular}{|c|c|c|c|c|}
\hline & Augusto & Tiberio & Calígula & $\begin{array}{l}\text { Indeter- } \\
\text { minadas }\end{array}$ \\
\hline $\begin{array}{lllllllll}\text { Bilbilis } & \ldots & \ldots & \ldots & \ldots & \ldots & \ldots & \ldots & \ldots\end{array}$ & & 2 & & \\
\hline $\begin{array}{lllllll}\text { Caesaraugusta } & \ldots & \ldots & \ldots & \ldots & \ldots & \ldots\end{array}$ & 10 & 5 & 3 & \\
\hline $\begin{array}{llllllll}\text { Calagurris } & \ldots & \ldots & \ldots & \ldots & \ldots & \ldots & \ldots\end{array}$ & 4 & 2 & & \\
\hline 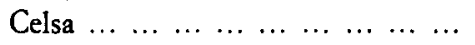 & 8 & & & \\
\hline $\begin{array}{ccccccccc}\text { Clunia } & \ldots & \ldots & \ldots & \ldots & \ldots & \ldots & \ldots & \ldots\end{array}$ & & 56 & & \\
\hline $\begin{array}{lllllllll}\text { Emerita } & \ldots & \ldots & \ldots & \ldots & \ldots & \ldots & \ldots & \ldots\end{array}$ & & 2 & & \\
\hline $\begin{array}{lllllllll}\text { Ercavica } & \ldots & \ldots & \ldots & \ldots & \ldots & \ldots & \ldots & \ldots\end{array}$ & 1 & & 2 & \\
\hline $\begin{array}{llllllll}\text { Graccurris } & \ldots & \ldots & \ldots & \ldots & \ldots & \ldots & \ldots\end{array}$ & & 2 & & \\
\hline $\begin{array}{llllllllll}\text { Ilici } & \ldots & \ldots & \ldots & \ldots & \ldots & \ldots & \ldots & \ldots & \ldots\end{array}$ & 2 & & & \\
\hline $\begin{array}{llllllllll}\text { Osca } & \ldots & \ldots & \ldots & \ldots & \ldots & \ldots & \ldots & \ldots & \ldots\end{array}$ & & 1 & & \\
\hline $\begin{array}{lllllllll}\text { Turiaso } & \ldots & \ldots & \ldots & \ldots & \ldots & \ldots & \ldots & \ldots\end{array}$ & 2 & 3 & & \\
\hline $\begin{array}{llllllll}\text { Noroeste } & \ldots & \ldots & \ldots & \ldots & \ldots & \ldots & \ldots\end{array}$ & 4 & & & \\
\hline $\begin{array}{llllllll}\text { Nemausus } & \ldots & \ldots & \ldots & \ldots & \ldots & \ldots & \ldots\end{array}$ & 2 & & & \\
\hline $\begin{array}{llllllll}\text { Lugdunum } & \ldots & \ldots & \ldots & \ldots & \ldots & \ldots & \ldots\end{array}$ & 3 & & & \\
\hline $\begin{array}{lllllllll}\text { Roma } & \ldots & \ldots & \ldots & \ldots & \ldots & \ldots & \ldots & \ldots\end{array}$ & 2 & & 1 & \\
\hline Total $\ldots \ldots \ldots \ldots \ldots$ & 38 & 73 & 6 & \\
\hline $\begin{array}{llllllll}\text { Porcentajes } & \ldots & \ldots & \ldots & \ldots & \ldots & \ldots & \ldots\end{array}$ & 32,48 & 62,39 & 5,13 & \\
\hline
\end{tabular}




\section{CLUNIA}

\section{Aporte porcentual por cecas}

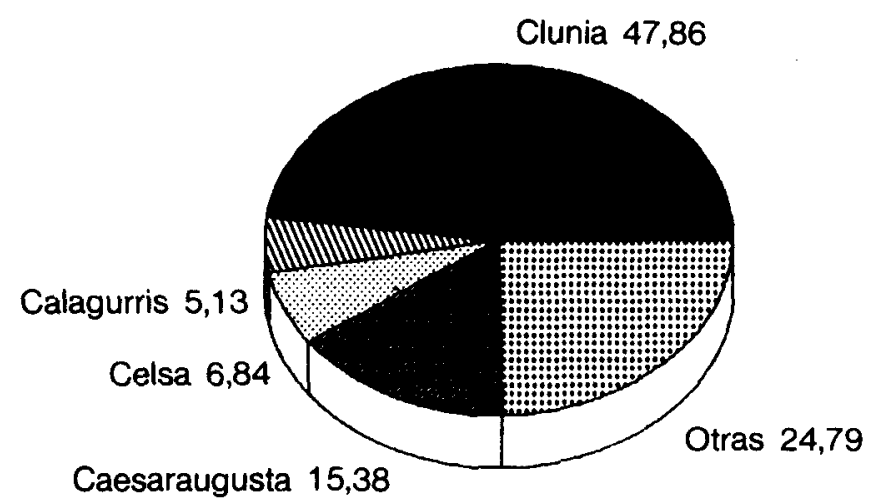

\section{MESETA LEONESA}

Las monedas proceden de la ciudad de Astorga y su entorno, así como del territorio meseteño de la provincia de León. La mayoría del numerario de Astorga tiene su origen en los hallazgos realizados durante las remociones del suelo urbano. Los datos han sido extraídos de las publicaciones de Delibes (1975), Fernández Aller (1978), y Mañanes (1982). Fernández Aller puntualiza que los ejemplares que recoge son de procedencia incierta, aunque puede presuponerse que muchos de ellos fueron encontrados en las comarcas aledañas al Museo Arqueológico de León ${ }^{7}$. Incluimos en nuestro inventario los numismas hispanolatinos que Mañanes presenta en el Apéndice (pp. 278283), a excepción de las piezas núm. 11 (p. 279), núm. 15 (p. 280) y núm. 23 (p. 281), pues el autor considera que podrían identificarse con otras ya descritas en su catálogo general ${ }^{8}$. En el cuadro 4 hemos recogido y clasificado las monedas de esta región.

Todos los hallazgos de estos conjuntos se presentan en el cuadro 5 desglosados por cecas, figurando también allí el porcentaje de aportación de cada ceca al total de cada grupo. En el cuadro 6 se recoge el porcentaje de presencia de los talleres que suponen más de un 5 por 100 del total, dentro

7 Fernández Aller (1978), p. 9.

8 Mañanes (1982), p. 278, nota 6. 


\section{CUADRO 4}

\section{Meseta Leonesa}

\begin{tabular}{|c|c|c|c|c|}
\hline & Augusto & Tiberio & Caligula & $\begin{array}{l}\text { Indeter- } \\
\text { minadas }\end{array}$ \\
\hline 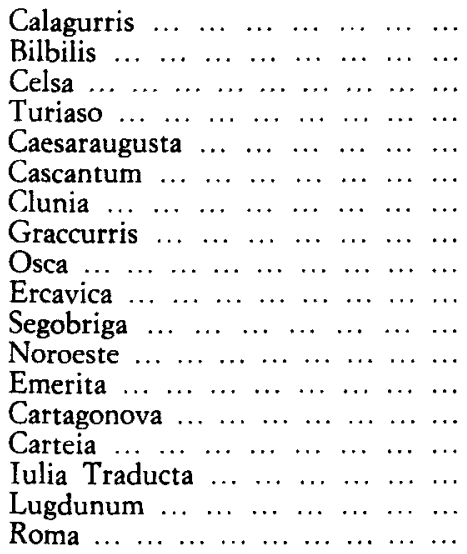 & $\begin{array}{l}1 \\
2 \\
2 \\
1 \\
1 \\
1\end{array}$ & $\begin{array}{r}11 \\
1 \\
1 \\
12 \\
\\
5 \\
4 \\
3\end{array}$ & 1 & 1 \\
\hline 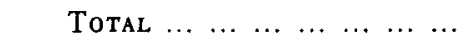 & 49 & 44 & 8 & 1 \\
\hline $\begin{array}{lllllllll}\text { Porcentajes } & \ldots & \ldots & \ldots & \ldots & \ldots & \ldots & \ldots\end{array}$ & 48,04 & 43,14 & 7,84 & 0,98 \\
\hline
\end{tabular}

\section{LEON}

Aporte porcentual por cecas

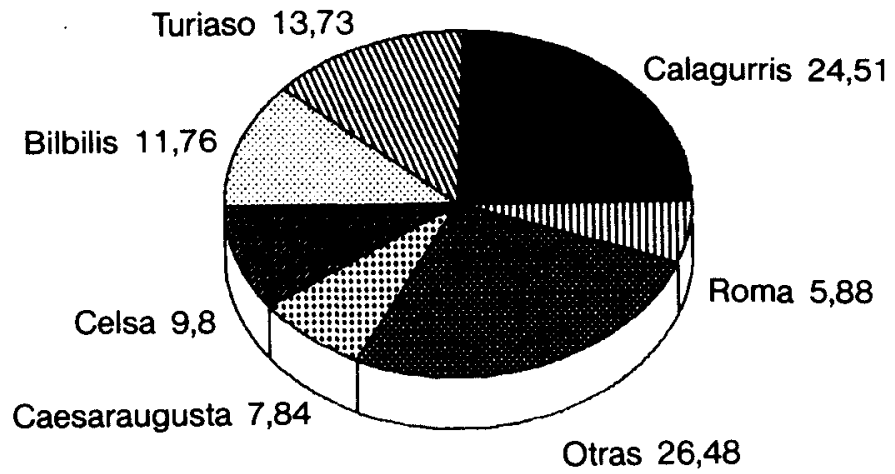


de cada conjunto. Finalmente, hemos elaborado cuatro gráficos de tarta, a partir de los datos que aparecen en el cuadro 6.

\section{CUADRO 5}

Procedencia de los hallazgos en cada conjunto

\begin{tabular}{|c|c|c|c|c|c|c|c|c|}
\hline & Jalón & $\%$ & Ebro & $\%$ & Clunia & $\%$ & León & $\%$ \\
\hline $\begin{array}{ccccc}\text { Clunia } & \ldots & \ldots & \ldots & \ldots\end{array}$ & 2 & 2,53 & 2 & 2,78 & 56 & 47,86 & 4 & 3,92 \\
\hline $\begin{array}{llll} & \\
\text { Calagurris } & \ldots & \ldots & \ldots\end{array}$ & 5 & 6,33 & 13 & 18,06 & 6 & 5,13 & 25 & 24,51 \\
\hline $\begin{array}{cccc}\text { Graccurris } & \ldots & \ldots & \ldots\end{array}$ & - & - & 1 & 1,39 & 2 & 1,71 & 3 & 2,94 \\
\hline Cascantum $\ldots \ldots$ & 2 & 2,53 & 2 & 2,78 & - & - & 5 & 4,90 \\
\hline Turiaso $\ldots \ldots \ldots \ldots$ & 4 & 5,06 & 1 & 1,39 & 5 & 4,27 & 14 & 13,73 \\
\hline $\begin{array}{llllll}\text { Bilbilis } & \ldots & \ldots & \ldots & \ldots\end{array}$ & 17 & 21,52 & 7 & 9,72 & 2 & 1,71 & 12 & 11,76 \\
\hline $\begin{array}{cccc}\text { Caesaraugusta } & \ldots & \ldots \\
\end{array}$ & 15 & 18,99 & 9 & 12,50 & 18 & 15,38 & 8 & 7,84 \\
\hline Celsa $\ldots \ldots \ldots \ldots \ldots$ & 9 & 11,39 & 5 & 6,94 & 8 & 6,84 & 10 & 9,80 \\
\hline Osca $\ldots \ldots \ldots \ldots$ & 3 & 3,80 & 3 & 4,17 & 1 & 0,85 & 1 & 0,98 \\
\hline $\begin{array}{lllll}\text { Ilerda } & \ldots & \ldots & \ldots & \ldots\end{array}$ & 1 & 1,27 & - & - & - & - & - & - \\
\hline $\begin{array}{ccccc}\text { Tarraco } & \ldots & \ldots & \ldots & \ldots\end{array}$ & 1 & 1,27 & - & - & - & - & - & - \\
\hline Noroeste $\ldots \ldots \ldots \ldots$ & - & - & - & - & 4 & 3,42 & 2 & 1,96 \\
\hline $\begin{array}{lllll}\text { Ercavica } \ldots & \ldots & \ldots & \ldots\end{array}$ & 3 & 3,80 & 1 & 1,39 & 3 & 2,56 & 1 & 0,98 \\
\hline Segobriga ......... & - & - & 1 & 1,39 & - & - & 2 & 1,96 \\
\hline Emerita $\ldots \ldots \ldots \ldots$ & 5 & 6,33 & 7 & 9,72 & 2 & 1,71 & 5 & 4,90 \\
\hline $\begin{array}{lllll}\text { Italica } & \ldots & \ldots & \ldots & \ldots\end{array}$ & . - & - & 1 & 1,39 & - & - & - & - \\
\hline Colonia Romula .... & 1 & 1,27 & - & - & - & 一 & - & - \\
\hline Colonia Patricia .... & - & - & 4 & 5,56 & - & - & - & - \\
\hline $\begin{array}{llllll}\text { Ilici } & \ldots & \ldots & \ldots & \ldots & \ldots\end{array}$ & - & - & - & - & 2 & 1,71 & - & - \\
\hline Cartagonova $\ldots \ldots \ldots$ & - & - & 3 & 4,17 & - & - & 1 & 0,98 \\
\hline $\begin{array}{ccccc}\text { Carteia } & \ldots & \ldots & \ldots & \ldots\end{array}$ & 5 & 6,33 & 3 & 4,17 & - & - & 1 & 0,98 \\
\hline Iulia Traducta ... ... & 3 & 3,80 & 4 & 5,56 & - & - & 1 & 0,98 \\
\hline $\begin{array}{llll}\text { Nemausus } & \ldots & \ldots & \ldots\end{array}$ & - & - & - & - & 2 & 1,71 & - & - \\
\hline $\begin{array}{llll}\text { Lugdunum } & \ldots & \ldots & \ldots\end{array}$ & - & - & 3 & 4,17 & 3 & 2,56 & 1 & 0,98 \\
\hline Roma $\ldots \ldots \ldots \ldots \ldots$ & 3 & 3,80 & 2 & 2,78 & 3 & 2,56 & 6 & 5,88 \\
\hline Total ....... & 79 & 100 & 72 & 100 & 117 & 100 & 102 & 100 \\
\hline Número de cecas ... & 16 & & 19 & & 15 & & 18 & \\
\hline
\end{tabular}




\section{CUADRO 6}

Cecas con presencia superior al 5 por 100

\begin{tabular}{|c|c|c|c|}
\hline Cecas & Valle del Ebro & Cecas & Meseta Leonesa \\
\hline 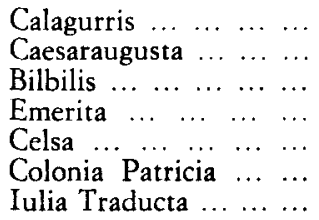 & $\begin{array}{r}18,06 \\
12,50 \\
9,72 \\
9,72 \\
6,94 \\
5,56 \\
5,56\end{array}$ & 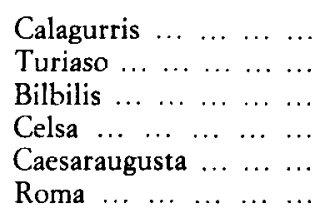 & $\begin{array}{r}24,51 \\
13,73 \\
11,76 \\
9,80 \\
7,84 \\
5,88\end{array}$ \\
\hline TOTAL .......... & 68,06 & Total .......... & 73,52 \\
\hline Resto cecas $\ldots \ldots \ldots \ldots$ & 31,94 & Resto cecas ... . . . . . . & 26,48 \\
\hline Cecas & Valle del Jalón & Cecas & Clunia \\
\hline 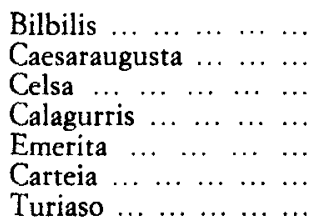 & $\begin{array}{r}21,52 \\
18,99 \\
11,39 \\
6,33 \\
6,33 \\
6,33 \\
5,06\end{array}$ & $\begin{array}{rrrrrr}\text { Clunia } & \ldots & \ldots & \ldots & \ldots & \ldots \\
\text { Caesaraugusta } & \ldots & \ldots & \ldots \\
\text { Celsa } & \ldots & \ldots & \ldots & \ldots & \ldots \\
\text { Calagurris } & \ldots & \ldots & \ldots & \ldots \\
& & & & & \\
\text { Total } & \ldots & \ldots & \ldots & \ldots\end{array}$ & $\begin{array}{r}47,86 \\
15,38 \\
6,84 \\
5,13\end{array}$ \\
\hline Total .......... & 75,95 & & \\
\hline Resto cecas ... ... . . . . & 24,05 & Resto cecas ... . . . . . & 24,79 \\
\hline
\end{tabular}

\section{ANALISIS ESTADISTICO DE LA COMPOSICION POR CECAS: ASOCIACION ENTRE CIRCULACIONES MONETARIAS}

Las pruebas y conceptos estadísticos que utilizaremos a continuación pueden encontrarse en las obras de Viedma (1976), Cuadras et al. (1984), San Martín, Espinosa y Pedreira (1984), y Villaronga (1985), así como en otros muchos manuales.

Intentaremos ver ahora cuál es la relación entre los abastecimientos de numerario correspondientes a los cuatro conjuntos que estamos analizando. Es decir, en qué medida se parece el perfil de la circulación monetaria de cada uno de estos lugares al de cada uno de los otros, considerando los 
aportes de moneda por cecas (no por emperadores) que evidencian las muestras de que disponemos. En nuestro análisis no incluiremos la variable tiempo (que vendría definida en sus valores por la fecha de producción de las emisiones o, a nivel más general, por el reinado en que fueron efectuadas), y ello por dos razones 1) La mayoría de las cecas, y especialmente las más importantes, están en funcionamiento durante todo el período analizado, cesando de emitir algunas en la fase final, que, como hemos comentado, abarcaría un corto número de años $(37.40 \mathrm{~d}$. C.). 2) La velocidad de circulación y la permanencia en la corriente monetaria de los numismas acuñados en el período que aquí contemplamos nos hacen pensar que las diferencias más importantes que se observan en las cronologías de estos conjuntos se deben a la diversidad de momentos en que esos territorios se incorporaron a la economía monetaria, puesto que el numerario precedente, aunque no habría sido retirado, seguiría circulando prioritariamente en las zonas donde llegó al poco de su producción, habiendo además disminuido su volumen por pérdida y desgaste. Por estas circunstancias se ha considerado inútil tener en cuenta el factor tiempo a la hora de aplicar análisis estadísticos.

A continuación vamos a utilizar pruebas que nos permitan determinar si dos muestras que se incluyen en los cálculos proceden de una misma población, presentando en caso afirmativo parecidas distribuciones de volumen de moneda por cecas. Si es así, podremos aceptar que el carácter del abastecimiento en ambas zonas de origen es muy similar, lo cual permitirá extraer conclusiones, que serían de otra índole si la hipótesis es rechazada. Para este propósito, trataremos cada uno de los conjuntos como una variable estadística, cuya información está categorizada en una escala nominal. La prueba más idónea al efecto sería el test $\mathrm{X}^{2}$ para $\mathrm{K}$ muestras. Sin embargo, existen inconvenientes que desaconsejan la adopción de esta técnica. En primer lugar, ese método de comparación de distribuciones sólo puede llevarse a cabo cuando son muy numerosas las observaciones en cada categoría, puesto que no más del 20 por 100 de las frecuencias esperadas pueden ser inferiores a 5 , y ninguna menor que 1 . Por otra parte, si estas condiciones no se cumplen es posible agrupar las clases contiguas hasta conseguir un número igual o superior a 5 observaciones. Pero ello, en este caso, desvirtuaria total o parcialmente los resultados, al tratarse de información que representa el número de piezas de una ceca hallado en una zona. ¿Cómo habrían de unirse los datos de las cecas? ¿Por la proximidad geográfica entre ellas? ¿Qué valor tendría la información así obtenida?

Por tanto, nos encontramos con la dificultad de que, con el escaso número de datos por taller y variable que tenemos, sería imposible cumplir los requisitos exigidos para la aplicación de la prueba de $\mathrm{X}^{2}$, salvo que se acumulase información, lo cual no es aconsejable por su pérdida de valor y 
la falta de criterios objetivos al respecto. En definitiva, y dada la escasez de las observaciones de que disponemos, recurriremos a la prueba de Kolmogorov-Smirnov, más eficiente que $\mathrm{X}^{2}$ para muestras pequeñas. Sin embargo, de este modo sólo podremos comparar las distribuciones de volumen de moneda por cecas entre parejas de variables. A la hora de efectuar los cálculos ha sido necesario tener en cuenta que los conjuntos no incluyen representación de las mismas cecas, existiendo piezas de algunos talleres sólo en uno de los dos. En estos casos, el valor adjudicado a esa ceca en la variable donde no está presente es 0 . Los resultados obtenidos de la aplicación de la prueba de Kolmogorov-Smirnov entre parejas de conjuntos han sido:

\begin{tabular}{|c|c|c|c|}
\hline & Ebro & Clunia & León \\
\hline 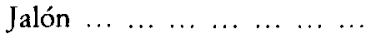 & 0,1737 & 0,4584 & 0,3354 \\
\hline $\begin{array}{llllllll}\text { Ebro } & \ldots & \ldots & \ldots & \ldots & \ldots & \ldots & \ldots\end{array}$ & & 0,4509 & 0,2565 \\
\hline $\begin{array}{lllllll}\text { Clunia } & . . & \ldots & \ldots & \ldots & \ldots & \ldots\end{array}$ & & & 0,4394 \\
\hline
\end{tabular}

Aquí hemos consignado, pues, la máxima diferencia entre las distribu ciones. Veamos cuáles son los valores críticos correspondientes a estas pruebas:

\begin{tabular}{|c|c|c|c|c|c|c|}
\hline & $J a l / E b$ & $\mathrm{Jal} / \mathrm{Clu}$ & Jal/Le & $\mathrm{Eb} / \mathrm{Clu}$ & Eb/Le & Clu/Le \\
\hline $\begin{array}{lllllll}\mathbf{D}_{0,05} & \ldots & \ldots & \ldots & \ldots & \ldots\end{array}$ & 0,2216 & 0,1980 & 0,2038 & 0,2037 & 0,2093 & 0,1842 \\
\hline $\begin{array}{cccccc}\mathrm{D}_{0,01} & \ldots & \ldots & \ldots & \ldots & \ldots\end{array}$ & 0,2737 & 0,2446 & 0,2518 & 0,2516 & 0,2586 & 0,2276 \\
\hline
\end{tabular}

Estos tests han puesto de relieve: 1) Que las muestras procedentes de los valles del Jalón y Ebro pertenecen a una misma población, puesto que evidencian distribuciones similares de los volúmenes de suministro por cecas. Es decir, que estos conjuntos se han extraído de áreas con igual alimentación monetaria, desde el punto de vista de la procedencia de las emisiones. Este es el resultado, a un nivel de significación del 5 por 100. 2) Las muestras del Valle del Ebro y la zona meseteña de León, podría aceptarse que proceden de una misma población, a un nivel de significación del 1 por 100; sus distribuciones son también, pues, similares. 3) El resto de las muestras proceden de poblaciones distintas, es decir, que las circulaciones monetarias 
que representan son diferentes en cuanto al perfil de su alimentación de numerario por cecas.

\section{COMENTARIO}

\section{A) Composición del numerario por emperadores}

Observemos, primeramente, la proporción de monedas por emperador que se aprecia en cada uno de estos conjuntos. En el Valle del Ebro, tempranamente romanizado, se evidencia un notable predominio de las emisiones augusteas, con presencia mucho menor de acuñaciones de Tiberio y escasas monedas de Calígula. El panorama es muy similar en el Valle del Jalón, aunque en esta zona el volumen proporcional de ejemplares de Augusto ha disminuido por el aumento de los aportes de piezas de Tiberio y Calígula, a lo que ha de sumarse que algunos bronces bilbilitanos son de cronología indeterminada, si bien inscrita dentro de los límites temporales que aquí se contemplan.

En Clunia dominan claramente las acuñaciones de Tiberio. Las causas de ello son perfectamente conocidas. Como ha señalado Gurt, es durante el reinado de este emperador cuando se produce uno de los momentos de mayor impulso constructivo en la ciudad, iniciándose entonces en Clunia, que se constituye como ceca, la verdadera circulación monetaria ${ }^{9}$.

Por lo que respecta a la zona leonesa, se aprecia que la proporción de emisiones augusteas y tiberianas es muy similar, mientras que la presencia de monedas de Calígula es elevada, en comparación con su volumen en los demás conjuntos. Evidentemente, el acceso a la economía monetaria debió ser, en esta zona, mucho más tardío que en las regiones mediterráneas o en el Valle del Ebro, a lo cual contribuiría también la escasa vida urbana que existió en este territorio durante buena parte del período cronológico estudiado. Domergue piensa que hasta la terminación de las guerras cántabras, en 19 a. C., apenas debieron de organizarse ni explotarse económicamente las zonas del noroeste peninsular ${ }^{10}$; por otra parte, parece que las explotaciones mineras comenzaron, con cierta intensidad, a finales del reinado de Augusto o a principios del de Tiberio, en los territorios situados al oeste de Astorga. En resumen, que tanto la tardía pacificación del área leonesa como el retraso de su desarrollo urbano y económico tuvieron como consecuencia la inexistencia de una circulación monetaria estable hasta un momento que cabría situar en el reinado de Tiberio e, incluso, comienzos del de Calígula.

Q Gurt (1985), pp. 74 y 233.

10 Domergue (1986), pp. 32-33. 


\section{B) Composición del numerario por cecas}

En cuanto al aporte de numerario por talleres, el panorama es diferente por conjuntos, aunque se evidencian algunas constantes de interés. Calagurris es la ceca mayoritaria en Ebro y León, y se encuentra entre los talleres más representados en Jalón y Clunia. Caesaraugusta y Celsa muestran aportes superiores al 5 por 100 del total en los cuatro conjuntos, y las monedas de la primera ciudad son las que ocupan el segundo lugar en grado de presencia en las circulaciones del Valle del Ebro, Jalón y Clunia. También los ejemplares de Bilbilis tienen importancia en las alimentaciones monetarias del Jalón - donde son mayoritarios-, Ebro y León.

Por lo que respecta a la estructura interna de los conjuntos, en el Valle del Ebro se aprecia un claro dominio de las cecas situadas en estos territorios, a la vez que destaca el peso que tienen, dentro de esta zona, las acuñaciones del sur peninsular (Emerita, Colonia Patricia, Iulia Traducta, Cartagonova y Carteia). Inicialmente pensamos que esa notoria presencia de talleres meridionales podía deberse, quizá, al origen extraño de parte de los materiales del Museo Provincial de Logroño. Sin embargo, las monedas de Emerita se encuentran también, según nuestros datos, en el Valle del Jalón, donde han aparecido igualmente bronces de Carteia y Iulia Traducta. Por tanto, no podemos desechar cierta influencia de la amonedación de algunas ciudades del sur hispano en los territorios dominados por el Ebro y sus principales afluentes.

En el Valle del Jalón es mayoritaria la ceca local, Bilbilis; son también muy importantes los aportes de los núcleos del Ebro medio, y tienen una presencia relativamente significativa, como hemos dicho, las amonedaciones del sur.

El volumen principal de emisiones en la zona leonesa lo constituyen las acuñaciones de Calagurris, siendo muy predominantes en la circulación monetaria del territorio las cecas del Valle del Ebro. También aquí se han hallado ejemplares emeritenses. En Clunia, casi la mitad del numerario corresponde a la ceca local, y se encuentran fuertemente representados los talleres del Ebro medio, mientras que aparecen pocas emisiones de las ciudades meridionales de la Península.

\section{C) Conclusiones}

En general, podemos concluir que la circulación monetaria de las zonas que analizamos, por lo que respecta a la procedencia del numerario, se encuentra ampliamente dominada por los talleres del Valle Medio del Ebro. 
La gran dispersión de estas emisiones ha sido señalada en repetidas ocasiones, con especial referencia a las acuñaciones de Caesaraugusta y Calagurris, seguidas de las monedas de Celsa y Bilbilis ". La producción de estas ciudades se difunde ampliamente hacia el oeste y noroeste peninsulares, rebasando los limites locales de forma evidente. Esta circunstancia se ha puesto en relación con la ubicación estratégica de los núcleos emisores, que les permitía constituirse en avanzadilla de la romanización y en suministradores de numerario al ejército acantonado en los territorios noroccidentales de la Citerior ${ }^{12}$.

En cada uno de los conjuntos, el predominio de uno u otro taller se explica por una serie de circunstancias propias. Así, en el Valle del Jalón es Bilbilis, la ceca local, quien proporciona el mayor número de monedas, seguida de las emisiones de la cercana Caesaraugusta. En el conjunto del Valle del Ebro, el numerario más abundante corresponde también al taller de más intensa producción de esta zona, Calagurris. Igualmente, es perfectamente lógico que las emisiones de esta ceca, junto con las de otras dos ubicadas, como ella, en los territorios occidentales del Ebro medio, dominen la alimentación monetaria a la zona meseteña. Clunia se autoabastece de numerario en buena medida, aunque en esta ocasión ha de tenerse en cuenta que, el enorme predominio de los numismas de la ciudad dentro del conjunto, puede haber venido determinado porque éste se ha constituido a partir de los hallazgos efectuados en el núcleo urbano, y no en una amplia zona.

Hemos observado también que la incidencia de la amonedación de las ciudades meridionales tiene cierta importancia en los territorios del Ebro y Jalón, que es menor en León, y de muy escasa intensidad en Clunia. Y, finalmente, cabe señalar el carácter residual que tiene la presencia de los numerarios de Roma y las ciudades galas dentro de estos conjuntos. Esta circunstancia, paralela al absoluto predominio de las cecas hispanorromanas en la circulación peninsular, ha sido señalada ya en diversas ocasiones ${ }^{13}$. Sólo en León hemos hallado un volumen relativamente apreciable de acuñaciones de la capital del Imperio, y en la zona del Ebro se ha puesto de relieve cierta presencia, aunque poco importante, del numerario de Lugdunum. Por otra parte, nuestro estudio contradice la opinión expresada por algunos investigadores en el sentido de que la circulación monetaria de este momento tendría un carácter cerrado, es decir, que las cecas que inciden en un centro o zona son siempre las más cercanas geográficamente, o las que tienen comunicaciones más directas con ese territorio ${ }^{14}$. Como hemos visto, el peso del numerario de los talleres del sur es importante en la circulación del Valle del

"Beltrán y Beltrán (1980), p. 49.

12 Beltrán (1986), pp. 910-911.

13 Ripollés (1982), p. 521; Gurt (1985), p. 75.

14 Gurt (1985), p. 75. 
Ebro, y todavía se aprecia su influencia en la zona del Jalón, si bien ésta pierde rápidamente intensidad conforme nos desplazamos hacia el oeste de Hispania.

Por otro lado, las pruebas estadísticas han puesto de relieve la relación, o falta de ella, entre estas cuatro circulaciones monetarias, proporcionando unos resultados que, a nuestro entender, presentan un panorama lógico y coherente. Partiendo de ellos, podemos aceptar que la alimentación monetaria a los valles del Ebro y Jalón debió ser muy similar entre 27 a. C. y 41 d. C., constituyendo ambas zonas un único territorio en cuanto a las cecas que las abastecían de numerario. A su vez, las áreas del Ebro y León, al parecer, presentan también relación en cuanto a la naturaleza de sus circulaciones monetarias, pero no sucede lo mismo entre la alimentación de los territorios leoneses y la del Valle del Jalón. Es decir, que se aprecia una evolución de Este a Oeste, puesto que el numerario que circula en el Jalón tiene estructura similar al de la zona del Ebro, y este último, del cual se nutre en buena parte la Meseta, presenta un perfil muy semejante al de León; pero la composición de la circulación monetaria en el Jalón es distinta a la de León.

Por último, Clunia parece aislarse del resto de los conjuntos, debido, sin duda, al enorme peso de las emisiones de la propia ciudad dentro del numerario circulante en ella.

\section{BIBLIOGRAFIA}

Beltrán Lloris, Francisco (1986): “Sobre la función de la moneda ibérica e hispanoromana", Estudios en bomenaje al doctor Antonio Beltrán Martinez, Zaragoza, pp. 889-914.

Beltrán Lloris, Miguel, y Beltrán Lloris, Francisco (1980): «Numismática hispanoromana de la Tarraconense», Numisma, 162-64, Alicante (Madrid), pp. 9-98.

Cundras, Carles M., et al. (1984): Fundamentos de Estadistica Aplicación a las Ciencias Humanas, Barcelona, PPU.

Delibes DE CASTRO, Germán (1975): Colección arqueológica «Don Eugenio Merino» de Tierra de Campos, León, Caja de Ahorros de León.

Domergue, Claude (1986): «Dix-huit ans de recherche (1968-1986) sur les mines d'or romaines du nord-ouest de la Péninsule Ibérique», Actas del I Congreso Internacional "Astorga Romana», Astorga, vol. II, pp. 7-101.

Domínguez, Almudena, y Galindo, María Pilar (1984): «Hallazgos numismáticos en el término de Calatayud», Gaceta Numismática, 74-75, Barcelona, pp. 63-103.

Elorza, Juan Carlos (1974): «Numismática Antigua en la provincia de Alava», Estudios de Arqueología Alavesa, 6, Vitoria, pp. 183-218.

Fernández Aller, María Carmen (1978): Epigratía y Numismática Romanas en el Museo Arqueológico de León, León, Colegio Universitario de León.

Galve, Pilar, y ANDrés, Sebastián (1984): «Hallazgos numismáticos en el yacimiento romano de Varea", Actas del I Symporium de Historia de Calaborra, Madrid, pp. 69-76. 
Gurt Esparraguera, Josep María (1985): Clunia III. Hallazgos monetarios. La romanización de la Meseta Norte a través de la circulación monetaria en la ciudad de Clunia, Excavaciones Arqueológicas en España, núm. 145, Madrid, Ministerio de Cultura.

IIménez, María C.; Luezas, Rosa A.; Sáenz, Pilar, e Iradier, Eva (1985): «Hallazgos numismáticos en Varea en la campaña de 1983», XVII Congreso Nacional de Arqueologia, Logroño (Zaragoza), pp. 759-775.

Mañanes Pérez, Tomás (1982): Epigrafía y numismática de Astorga romana y su entorno, Salamanca, Universidad de Salamanca.

Martín-Bueno, Manuel (1971): "Un áureo y otros hallazgos monetarios en Bilbilis (Calatayudis, Pyrenae, 7, Barcelona, pp. 145-155.

- (1973-74): "Circulación monetaria en Bilbilis. Motivaciones económicas», Numisma, 120-131, Zaragoza (Madrid), pp. 151-154.

- (1974): «Numismática antigua en el Museo Provincial de Logroño», Acta Numismática, IV, Barcelona, pp. 65-85.

Martín-Bueno, Manuel, y Redondo Veintemillas, Guillermo (1979): La Colección Numismática Dominguez del Museo de Calatayud, Calatayud, Centro de Estudios Bilbilitanos.

Medrano, Manuel: Díaz, María Antonia; Astiz, Laura, y Lizabe, Jorge (en prensa): «Las monedas ibéricas y romanas de Arcóbriga y Luzaga», Caesaraugusta, 66, Zaragoza.

Nieto Gallo. Gratiniano (1958): El Oppidum de Iruña (Alava), Vitoria.

Ripollés Ai.egre, Pere Pau (1982): La circulación monetaria en la Tarraconense medite. rránea, Valencia, Diputación Provincial de Valencia.

Sáenz de Buruaga, José Ántonio, y Sáenz de UrTuri, Francisca (1986): «Nuevos tipos monetales localizados en Alava», Estudios de Arqueología Alavesa, 13, Vitoria, pp. $239-271$.

SÁENZ dE URTURI, Francisca (1978): «Nuevos hallazgos romanos en Alava: marcas de alfarero y monedas», Estudios de Arqueología Alavesa, 9, Vitoria, pp. 293-299.

San Martín, R.; Espinosa, L., y Pedreira, L. F. (1984): Psicoestadística II, Universidad Autónoma de Madrid.

Ugartechea, José Miguel (1967): «Notas sobre el yacimiento de Salbatierrabide (Vitoria)», Estudios de Arqueología Alavesa, 2, Vitoria, pp. 101-118.

Viedma, Juan A. (1976): Biocstadistica, Madrid.

Villaronga, Leandre (1985): Estadistica Aplicada a la Numismática, Barcelona, Asociación Numismática Española. 
Denominación actual de los lugares donde se ubicaron las ciudades antiguas mencionadas en este trabajo

\begin{tabular}{|c|c|}
\hline Denominación antigua & Denominación actual \\
\hline Arcobriga & ¿Monreal de Ariza? (Zaragoza) \\
\hline Asturica & Astorga (León) \\
\hline Bilbilis & Calatayud (Zaragoza) \\
\hline Caesaraugusta & Zaragoza \\
\hline Calagurris & Calahorra (La Rioja) \\
\hline Cartagonova & Cartagena \\
\hline Carteia & Torre de Cartagena (San Roque, Cádiz) \\
\hline Cascantum & Cascante (Navarra) \\
\hline Celsa & ¿Velilla de Ebro? (Zaragoza) \\
\hline Clunia & $\begin{array}{l}\text { Restos ubicados entre Coruña del Conde } \\
\text { y Peñalba de Castro (Burgos) }\end{array}$ \\
\hline Colonia Patricia & Córdoba \\
\hline Colonia Romula & Sevilla \\
\hline Conimbriga & Condeixa-a-velha (Portugal) \\
\hline Emerita & Mérida (Badajoz) \\
\hline Ercavica & Cañaveruelas (Cuenca) \\
\hline Graccurris & Alfaro (La Rioja) \\
\hline Ilerda & Lérida \\
\hline Ilici & Alcudia de Elche (Alicante) \\
\hline Insula Augusta & Ibiza \\
\hline Italica & Santiponce (Sevilla) \\
\hline Iulia Traducta & ¿Algeciras? (Cádiz) \\
\hline Legio & León \\
\hline Lucus & Lugo \\
\hline Lugdunum & Lyon (Francia) \\
\hline Nemausus & Nìmes (Francia) \\
\hline Noroeste & $\begin{array}{l}\text { Monedas cuyo origen estuvo, posiblemen } \\
\text { te, en la ciudad de Lugo }\end{array}$ \\
\hline Osca & Huesca \\
\hline Roma & Roma \\
\hline Segobriga & Cabeza del Griego (Saelices, Cuenca) \\
\hline Tarraco & Tarragona \\
\hline Turiaso & Tarazona (Zaragoza) \\
\hline Varea & Logroño (La Rioja) \\
\hline
\end{tabular}

